\title{
硼氮嵌入的双重[7]螺烯
}

\author{
刘豫健王朝晖* \\ (清华大学化学系 北京 100084)
}

\section{B,N-Embedded Double Hetero[7]helicenes}

\author{
Liu, Yujian Wang, Zhaohui* \\ (Department of Chemistry, Tsinghua University, Beijing 100084)
}

利用手性物质的光学响应可构建高效手性光电子 器件, 例如圆偏振发光器件和探测器等 ${ }^{[1,2]}$. 螺烯作为具 有固有螺旋手性的邻位稠合多环芳香化合物, 其独特的 螺旋 $\pi$-共轭电子结构引起了极大的研究兴趣 ${ }^{[3]}$. 然而, 低阶的纯碳螺烯分子材料的不对称因子和荧光量子效 率普遍较低, 且主要在紫外光区表现出手性光学响应 ${ }^{[4]}$, 限制了其在上述手性光电子器件中的广泛应用. 目前, 文献中报道了通过 $\pi$-体系扩展 ${ }^{[5]}$ 、多重螺烯构建 ${ }^{[6]}$, 以 及杂原子掺杂 ${ }^{[7]}$ 等策略, 开发出了丰富的螺烯分子体系, 成功拓展了其手性光学响应范围, 但绝大多数分子的不 对称因子仍低于 $10^{-2}$. 如何通过分子理性设计开发出具 有高效手性光学性能的新型螺烯分子, 是该领域面临的 重大挑战.

近年来, B- $\mathrm{N}$ 共价掺杂以及 $\mathrm{B} \rightarrow \mathrm{N}$ 配位掺杂为 $\pi$-共 轭分子体系光电性能的改变提供了新的途径 ${ }^{[8-9]}$. 近日, 南开大学化学学院王小野课题组 ${ }^{[10]}$ 通过简单高效的两 步法将 $\mathrm{B}$ 和 $\mathrm{N}$ 原子以非键合的方式引入到芳香体系中, 合成了嗍氮杂双重[7]螺烯(图 1, 1a 1c). 研究人员通过 核磁共振波谱(NMR)和单晶 $\mathrm{X}$ 射线衍射确证了该螺烯 分子的对映异构体构型. 同时, 密度泛函理论(DFT)计 算表明了其异构化能垒高达 $282.69 \mathrm{~kJ} / / \mathrm{mol}$, 实验进一 步证实对映异构体在 $300{ }^{\circ} \mathrm{C}$ 加热 $3 \mathrm{~h}$ 后, 其构型仍保持 不变.

光谱数据表明分子的紫外 - 可见吸收光谱延伸到了 $700 \mathrm{~nm}$, 这得益于硼氮原子在分子内的推拉电子效应, 使得这类螺烯分子在 300 700 nm 区域内表现出较强的 手性光学响应, 吸收不对称因子 $g_{\text {abs }}$ 达到了 $0.033(\lambda=$ $502 \mathrm{~nm})$, 为目前螺烯衍生物在可见光区域内的吸收不 对称因子的最高纪录(图 2).

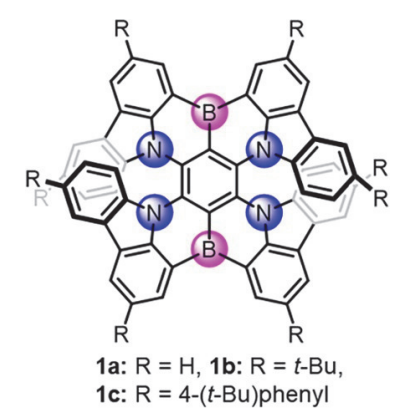

图 1 化合物 $1 \mathrm{a} \sim 1 \mathrm{c}$ 的结构

Figure 1 Structures of compound $\mathbf{1 a} \sim \mathbf{1 c}$

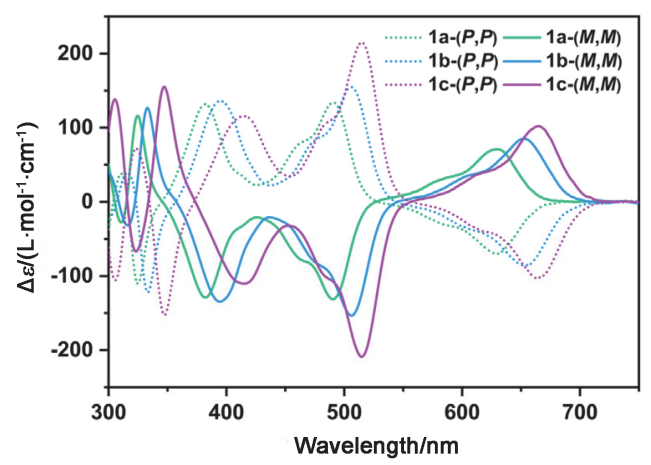

图 2 化合物 $1 \mathrm{a} \sim 1 \mathrm{c}$ 的圆二色光谱(于 $1 \times 10^{-5} \mathrm{~mol} / \mathrm{L}$ 二氯甲 烷溶液)

Figure $2 \mathrm{CD}$ spectra of $\mathbf{1 a} \sim \mathbf{1 c}$ (in $1 \times 10^{-5} \mathrm{~mol} / \mathrm{L} \mathrm{CH}_{2} \mathrm{Cl}_{2}$ solution)

通过含时密度泛函理论(TD-DFT)计算, 研究人员 将化合物 $1 \mathrm{a} \sim 1 \mathrm{c}$ 的具有最大吸收不对称因子的吸收峰 归属于 $\mathrm{S}_{0} \rightarrow \mathrm{S}_{2}$ 跃迁, 该跃迁主要对应于 $\mathrm{HOMO} \rightarrow$ LUMO +1 跃迁. 研究人员进一步通过理论计算分别获 得了 $\mathrm{S}_{0} \rightarrow \mathrm{S}_{1}$ 和 $\mathrm{S}_{0} \rightarrow \mathrm{S}_{2}$ 跃迁对应的电跃迁偶极矩 $\mu$ 、磁跃

* Corresponding author. E-mail: wangzhaohui@mail.tsinghua.edu.cn. Published online November 30, 2021. 
迁偶极矩 $m$ 及其两者之间的夹角 $\theta$. 根据公式 $g=$ $4|m| \cos \theta /|\mu|$, 在两种跃迁状态下, $\mu$ 和 $m$ 保持平行或反平 行, $\cos \theta$ 为最大值 \pm 1 , 而 $\mathrm{S}_{0} \rightarrow \mathrm{S}_{2}$ 跃迁却具有较小的 $\mu$ 值 和较大的 $m$ 值, 有助于获得更高的 $g$ 值. 这主要是非键 合的嗍氮原子引起的多重共振效应导致前线轨道分离 的结果, HOMO 与 $\mathrm{LUMO}+1$ 之间的分离程度更加明显, 较小的轨道重叠降低了跃迁的 $|\mu|($ 图 3).

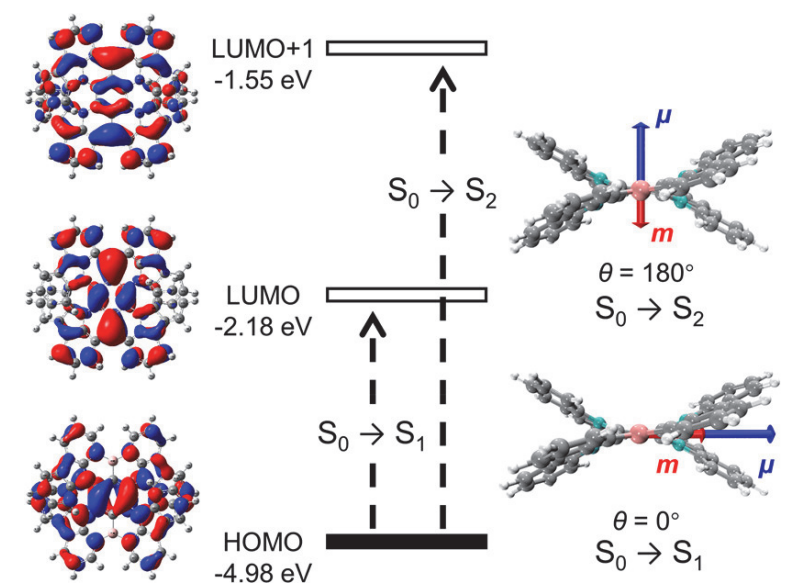

图 3 TD-DFT 计算得到的 1a- $(P, P)$ 的电跃迁偶极矩 $(\mu)$ 和磁跃 迁偶极矩 $(m)$

Figure 3 TD-DFT calculated results of 1a- $(P, P)$ with electric $(\mu)$ and magnetic $(m)$ transition dipole moments

轨道分离的结果同时也使得这些螺烯分子的单线 态-三线态能级差 $\left(\Delta E_{\mathrm{ST}}\right)$ 较小, 导致了热活化延迟苂光 (TADF)特性. 稳态和瞬态荧光光谱测试表明, 其具有接 近 $100 \%$ 的苂光量子效率, 瞬时寿命和延时寿命分别在 纳秒和微秒级. 利用分子的 TADF 特性可获得高效荧光 发光材料, 将手性中心与发光中心有机统一并最大化,
是实现螺烯分子体系高效圆偏振发光(CPL)的重要途径. 测试表明，该螺烯分子的圆偏振发光的不对称因子 $\left(g_{\text {lum }}\right)$ 可达 $0.002, \mathrm{CPL}$ 亮度 $\left(B_{\mathrm{CPL}}=\varepsilon \times \Phi_{\mathrm{F}} \times g_{\text {lum }} / 2\right)$ 达 40.0 $\mathrm{L}^{-1} \cdot \mathrm{mol}^{-1} \cdot \mathrm{cm}^{-1}$, 是一类优异的圆偏振发光材料.

手性分子的手性光学响应, 特别是圆偏振发光是构 建高效手性光电子器件的基础. 兼具高发光不对称因子 和高荧光量子效率的圆偏振发光材料的开发对推动其 实际功能应用具有重要意义. 通过理性设计调控分子的 $\mu 、 m$ 以及两者之间的夹角 $\theta$, 进而平衡发光不对称因子 和苂光量子效率，有望最终获得圆偏振发光性能的突 破. 因此，对于螺烯分子材料，我们认为首先应提高其 荧光量子效率，在此基础上，通过杂原子引入、分子对 称性的调控、多重度以及 $\pi$-体系扩展等方式获得发光不 对称因子的大幅度提升.

\section{References}

[1] Gong, Z.-L.; Zhu, X.; Zhou, Z.; Zhang, S.-W.; Yang, D.; Zhao, B.; Zhang, Y.-P.; Deng, J.; Cheng, Y.; Zheng, Y.-X.; Zang, S.-Q.; Kuang, H.; Duan, P.; Yuan, M.; Chen, C.-F.; Zhao, Y. S.; Zhong, Y.-W.; Tang, B. Z.; Liu M. Sci. China Chem. 2021, 64, 2060.

[2] Yang, Y.; da Costa, R. C.; Fuchter, M. J.; Campbell, A. J. Nat. Photon. 2013, 7, 634.

[3] Mori, T. Chem. Rev. 2021, 121, 2373.

[4] Shen, Y.; Chen, C.-F. Chem. Rev. 2012, 112, 1463.

[5] Qiu, Z.; Ju, C.-W.; Frédéric, L.; Hu, Y.; Schollmeyer, D.; Pieters, G.; Müllen, K.; Narita, A. J. Am. Chem. Soc. 2021, 143, 4661.

[6] Liu, B.; Böckmann, M.; Jiang, W.; Doltsinis, N. L.; Wang, Z. J. Am. Chem. Soc. 2020, 142, 7092.

[7] Dhbaibi, K.; Favereau, L.; Crassous, J. Chem. Rev. 2019, 119, 8846.

[8] Wang, X.-Y.; Zhuang, F.-D.; Wang, R.-B.; Wang, X.-C.; Cao, X.-Y.; Wang, J.-Y.; Pei, J. J. Am. Chem. Soc. 2014, 136, 3764.

[9] Zhao, R.; Liu, J.; Wang, L. Acc. Chem. Res. 2020, 53, 1557.

[10] Li, J.-K.; Chen, X.-Y.; Guo, Y.-L.; Wang, X.-C.; Sue, A. C.-H.; Cao, X.-Y.; Wang, X.-Y. J. Am. Chem. Soc. 2021, 143, 17958. 